\title{
An Efficient User-Oriented Clustering of Web Search Results
}

\author{
Keke Cai, Jiajun Bu, and Chun Chen \\ Deptartment of Computer Science, Zhejiang University, \\ Hangzhou, China, 310027 \\ \{caikeke, bjj, chenc\}@zju.edu.cn
}

\begin{abstract}
As a featured function of search engine, clustering display of search results has been proved an efficient way to organize the web resource. However, for a given query, clustering results reached by any user are totally identical. In this paper, we explored a user-friendly clustering scheme that automatically learns users' interests and accordingly generates interest-centric clustering. The basis of this personal clustering is a keyword based topic identifier. Trained by users' individual search histories, the identifier provides most of personal topics. Each topic will be the clustering center of the retrieved pages. The scheme proposed distinguishes the functionality of clustering from that of topic identification, which makes the clustering more personal and flexible. To evaluate the proposed scheme, we experimented with sets of synthetic data. The experimental results prove it an effective scheme for search results clustering.
\end{abstract}

\section{Introduction}

For the overwhelming information on Internet, conventional keyword-based Internet search usually return hundreds of thousands of retrieved results, most of which are irrelevant to what enquired. To solve this problem, automatic clustering technique has been proposed. Its main idea is to group retrieved pages by common topics, which is usually evaluated by the relevance among pages returned. Some schemes [1], [2] compare the direct or indirect hyperlinks of pages to gauge their relevance, whereas others, such as [3], utilize common keywords contained in different pages. These methods enable effective identification of retrieved results, however most of them rely particularly on retrieved web pages themselves to cluster. Consequently, for a given query the clustering results are always static.

Recently, user-oriented personal service has been widely deployed in the domain of search engine. A clustering scheme called content ignorant was invented [4], [5], which performs the clustering of queries beforehand for pages clustering to build upon. Though it provides a dynamic and rapid response for web surfing, its expansibility restrains because it can only establish mappings between queries and URLs that have already been preprocessed. In this paper, we explore a user-friendly clustering scheme that automatically learns user' interests and simultaneously generates interestcentric clustering. Differed from conventional pages clustering schemes, our clustering is designed to make the clustering more individual but not to provide user the most detailed clustering. The basis of this personal clustering is a keyword-based topic identifier, which is fully trained by user's individual search histories. When a 
user submits a query to search engine, it will automatically generate a set of topics with individual characteristics, which will be utilized in later page clustering.

\section{Personal Clustering Scheme}

The proposed clustering model consists of three basic components: Personal Topic Identifier, Query Executor and Personal Clustering Generator. Personal topic identifier recognizes personal topics of each user; Query executor processes user's current query and returns retrieved web pages; Personal clustering generator constructs the final clustering display of the retrieved web pages. Among these, personal topic identifier is the kernel. It monitors and records user's behaviors, and then denotes each visited page as a weighted keyword vector. It is believed that users' interests may lead to frequent visit to web pages of specific topics, which correspondingly causes the frequent co-occurrence of specific keywords. In this paper, such frequent keyword sets are regarded as the personal topics.

Equation 1 represents weight computation of term $t_{j}$ to page $p_{i}$. It is a modification of TF-IDF weighting scheme [6]. Terms with weight more than system-predefined threshold will be selected as page-related keywords. Influence factor $\log \left(N / n_{i, j}\right)$ reflects the frequency of $t_{j}$ in the condition of all web pages. $f_{i, j}$ is the normalized frequency of term $t_{j}$ in web page $p_{i}$. Parameter $\alpha$ reflect the position of term $t_{j}$ in page $p_{i}$, and it is more than 1 if $t j$ appears in the title of $p_{i}$, otherwise it is 1.

$$
W_{i, j}=\alpha^{*} f_{i, j} * \log \left(N / n_{i, j}\right) .
$$

Considering the number restriction of clusters as well as the requirement of the minimal overlap among clusters, we modify the CLOSET+ [7] frequency mining algorithm to finish the process of personal topics identification. To avoid serious overlap between closed keyword sets, we refine the results set so that if two keyword sets are similar enough, they are emerged. Equation 2 is the similarity definition between two keywords set $k s_{1}$ and $k s_{2}$ by cosine distance.

$$
\operatorname{sim}\left(k s_{1}, k s_{2}\right)=k s_{1} \cdot k s_{2} /\left\|k s_{1}\right\|\left\|k s_{2}\right\| .
$$

Based on the identified clustering topics as well as the retrieved query results, the finial clustering can be easily realized through our defined keywords-based cluster identifier. For a flat set of clustering $C=\left\{\mathrm{C}_{1} \ldots C_{n}\right\}$, it consists of a set of rules $f_{i} \rightarrow C_{i}$, $\mathrm{i}=1 \ldots \mathrm{n} . f_{i}$ is a frequent keyword set, which represents the corresponding topic information of $C_{i}$. A web page $p$ is considered belonging to the $C_{i}$, if $p$ matches the rule of $f_{i} \rightarrow C_{i}$ Function $\operatorname{ovp}()$ in equation 3 measures the match degree between pages and clusters, and the domain of its value ranges between 0 and $1 . k p$ represents the keyword vector of page $p$. We cluster $p$ to $C_{i}$ if their overlap is no less than the predefined threshold minSim. It is possible that some retrieved cannot be mapped to any cluster. In our paper, all these pages will be assigned to a separated cluster.

$$
\operatorname{ovp}\left(p, C_{i}\right)=\left|k p \cap f_{i}\right| /\left|f_{i}\right| .
$$

Table 1 shows the personal precision of three kinds of queries under personal clustering $(P C)$ and normal clustering $(N C)$. For clustering $P C$ and $N C$, their personal 
precision Personal is defined as a cosine distance against $M C$, which is another clustering results obtained through manual definition. As show in Table 1, our personal clustering method presents satisfactory adaptability in various queries, and its personal precision averagely reach about 83 percent. It shows that this clustering method can perfectly capture any topic information mostly concerned by user, and accordingly form a set of user-topic related clusters.

$$
\operatorname{Personal}(A C)=\frac{1}{|M C|} \sum_{A C_{i} \in A C} \max _{A C_{i}}\left(\operatorname{sim}\left(M C_{j}, A C_{i}\right)\right) .
$$

Table 1. Personal precision comparisons

\begin{tabular}{|c|c|c|c|c|c|c|}
\hline Data & \multirow{2}{*}{$\begin{array}{c}\text { Pages } \\
\text { Source }\end{array}$} & \multicolumn{4}{c|}{ Clusters Number } & \multicolumn{2}{c|}{$\begin{array}{c}\text { Personal Precision } \\
\text { (percent) }\end{array}$} \\
\cline { 3 - 7 } & & $M C$ & $P C$ & $N C$ & $P C$ & $N C$ \\
\hline Q1 & 99 & 4 & 3 & 8 & 89.2 & 57.8 \\
\hline Q 2 & 465 & 6 & 8 & 16 & 82.1 & 34.8 \\
\hline Q 3 & 1220 & 9 & 7 & 27 & 79.8 & 10.5 \\
\hline
\end{tabular}

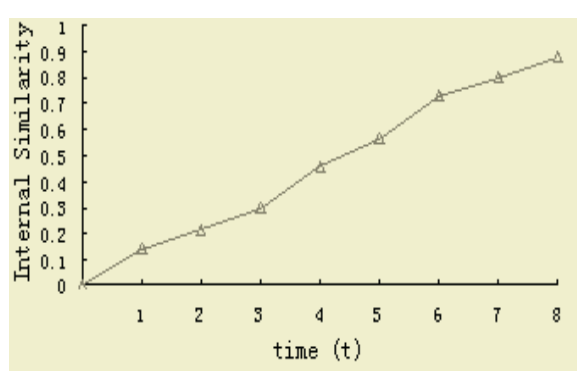

Fig. 2. Internal similarity of clusters

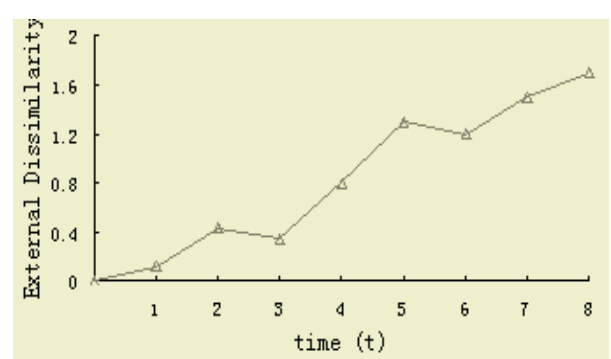

Fig. 3. External dissimilarity of clusters

$$
\text { internalSim }=\frac{1}{|C|} \sum_{C_{i} \in C} \frac{1}{\left|C_{i}\right|} \sum_{\substack{x \in C_{i} \\ y \in C_{i}}} \operatorname{sim}(x, y) .
$$

Fig. 2 and 3 show the quality of our clustering as client' query become more and more concentrated on some special topics. Internal similarity internalSim represents inner cohesiveness of each cluster, and external dissimilarity dep describes the independence of different clusters. The definition of dep is built upon the overlap of different clusters [8]. Given a page $p$ clustered to $C_{i}$, we define $O_{p}$ as the number of its occurrence in other clusters.

As shown in Fig. 2, the form of user query topics directly decides the clustering accuracy. With the gradual enrichment of user query topics information, the clustering 
becomes more and more accurate. Fig. 3 shows that this clustering can dynamically adapt user query, and capture any personal topics well and truly.

$$
\operatorname{dep}(C)=\frac{1}{|C|} \sum_{C_{i} \in C} \frac{1}{\left|C_{i}\right|} \sum_{p \in C_{i}}\left(O_{p}-1\right)
$$

\section{Conclusions}

This paper presents an efficient means for the personal clustering of retrieved web pages by learning users' interests with respect to their query histories, and further using this information to realize a meaningful clustering. With the enrichment of user history records, the clustering will pick and return even more accurate results.

\section{References}

1. Hou, J. and Zhang, Y., Utilizing Hyperlink Transitivity to Improve Web Page Clustering. Proceedings of the Fourteenth Australasian Database Conference. Adelaide, Australia (2003) 49-57

2. D. S. Modha and W. S. Spangler, Clustering hypertext with applications to web searching. In Proceedings of the 11th ACM Conference on Hypertext and Hypermedia. San Antonio, TX (2000) 143-152

3. Zhang Dong, Towards Web Information Clustering. PhD thesis. Southeast University, Nanjing, China (2002)

4. D. Beeferman and A. Berger, Agglomerative clustering of a search engine query log. In Proceedings of the Sixth ACM SIGKDD International Conference on Knowledge Discovery and Data Mining. Boston, MA (2000) 407-416

5. Mark Hansen, Elizabeth Shriver, Using navigation Data to Improve IR functions in the Context of Web Search. CIKM. (2001) 135-142

6. Ricardo Baeza-Yates, Berthier Ribeiro-Neto, Modern information retrieval. ACM Press. (1999)

7. J. Wang, J. Han, and J. Pei, CLOSET+:Searching for the Best Strategies for Mining Frequent Closed Itemsets. In Proc. 2003 ACM SIGKDD. Int. Conf. on Knowledge Discovery and Data Mining (KDD'03). Washington, D.C (2003)

8. F. Beil, M. Ester, and X. Xu., Frequent term-based text clustering. In Proc 8th Int. Conf. on Knowledge Discovery and Data Mining KDD. (2002) 